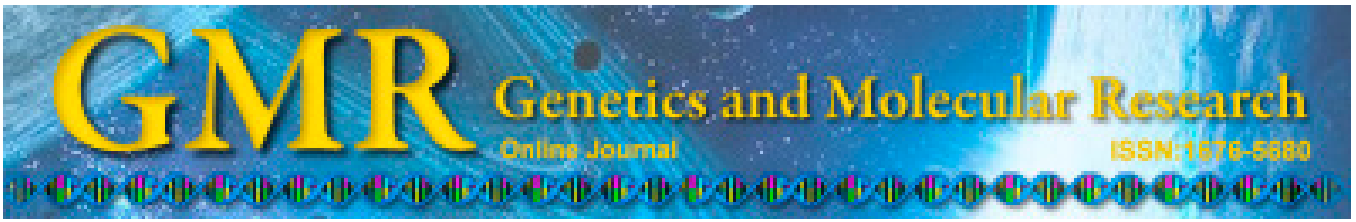

\title{
Autophagy in drug resistance of the multiple myeloma cell line RPMI8226 to doxorubicin
}

\author{
Y.-Z. Pan, X. Wang, H. Bai, C.-B. Wang, Q. Zhang and R. Xi \\ Department of Hematology, Lanzhou General Hospital, Lanzhou, \\ Gansu Province, China \\ Corresponding author: H. Bai \\ E-mail: panyaozhu_pyz@163.com
}

Genet. Mol. Res. 14 (2): 5621-5629 (2015)

Received August 8, 2014

Accepted December 4, 2014

Published May 25, 2015

DOI http://dx.doi.org/10.4238/2015.May.25.14

\begin{abstract}
We investigated the effect of autophagy on drug resistance of multiple myeloma (MM) to doxorubicin(DOX). ADOX-resistant MM cell line (RPMI8226/DOX) was developed by progressively increasing the DOX concentration gradient. The drug resistance index was determined using the MTT method. Transmission electron microscopy, anti-light chain 3-fluorescein isothiocyanate immunofluorescence, and Western blotting were used to detect autophagy of MM cells. Flow cytometry was applied to detect changes in apoptosis of RPMI8226/ DOX cells (stained with annexin-V/propidium iodide) caused by inhibition by hydroxychloroquine and 3-methyladenine on autophagy. The drug resistance index of RPMI8226/DOX to DOX was 10.8, and autophagy/lysosomal was clearly observed in RPMI8226/DOX cells under transmission electron microscopy, while immunofluorescence showed granular immunofluorescence in cells. Western blot analysis showed that light chain 3-II protein expression level was higher in RPMI8226/DOX cells than in RPMI8226/S cells. The apoptosis test showed that hydroxychloroquine or 3-methyladenine partially reversed the drug resistance of RPMI8226/DOX cells by inhibiting autophagy.
\end{abstract}


Activation of autophagy in MM cells may explain the drug resistance of myeloma.

Key words: Autophagy; Doxorubicin; Drug resistance; Myeloma; RPMI8226

\section{INTRODUCTION}

In eukaryotic cells, autophagy is a type of stress reaction that occurs following noxious stimuli such as malnutrition, hypoxia, and endoplasmic reticulum stress. In autophagy, cytoplasmic proteins and organelles that are injured or aged undergo degradation and are reconstructed as intracellular nutrients or macromolecules, such that the substances are recycled and organelles are renewed. Meticulous regulation of this process is very important for maintaining the balance of protein metabolism and intracellular homeostasis (Pan et al., 2011). Recent studies examining the relationship between autophagy and tumors have revealed some of the details of this process. However, few studies have examined the effect of autophagy on hematological malignancies.

Multiple myeloma (MM) belongs to the category of plasmacytoma. Therapy in most patients is favorable during early treatment. However, over time, patients experience recurrence and develop multidrug resistance. As one of the primary components of MM chemotherapy, resistance to doxorubicin (DOX) is quite common. In this study, we established a DOX-resistant MM cell line (RPMI8226/DOX) by progressively increasing the DOX concentration gradient and explored the effect of autophagy on drug resistance to provide a new therapeutic target.

\section{MATERIAL AND METHODS}

\section{Cell culture}

The human myeloma cell line RPMI8226 was supplied by the Pathology Department of The Fourth Military Medical University. Cells were grown in suspension in RPMI1640 medium supplemented with $10 \%$ fetal bovine serum, $1 \%$ (v/v) penicillin, $100 \mu \mathrm{g} / \mathrm{mL}$ streptomycin, and $1 \%(\mathrm{v} / \mathrm{v}) \mathrm{L}$-glutamine. Cells were incubated at $37^{\circ} \mathrm{C}$ in a $5 \% \mathrm{CO}_{2}$ humidified atmosphere and were passaged every 6 days.

\section{Drugs and reagents}

DOX injection was obtained from Pharmaceutical Co., Ltd., Shenzhen Wan Yue. Blue tetrazolium (MTT) and the rabbit anti-human $\beta$-actin polyclonal antibody were from Sigma (St. Louis, MO, USA). Rabbit anti-human light chain 3 (LC3) monoclonal antibody was from Abcam (Cambridge, UK).

\section{Induction of drug-resistant cell line RPMI8226/DOX}

DOX was added to the RPMI8226/sensitive (S) cell medium during the first medium change at a concentration of $10 \mathrm{nM}$ (approximately the $20 \%$ inhibitory concentration value) 
(Dalton et al., 1986). The medium was changed 3 times per week while progressively increasing DOX to a final concentration of $0.1 \mu \mathrm{M}$ at the 10th month. DOX concentration was maintained until its complete removal 1 week before the experiments.

\section{Drug sensitivity test using the MTT assay}

A total of $10 \mu \mathrm{M}$ RPMI8226/S cells and the same number of RPMI8226/DOX cells were seeded on 96 -well plates and incubated in RPMI 1640 at $37^{\circ} \mathrm{C}$ in a $5 \% \mathrm{CO}_{2}$ humidified atmosphere for $1 \mathrm{~h}$. Next, DOX was added to the cells in each group at concentrations of $1 \mathrm{nM}$, $10 \mathrm{nM}, 0.1 \mu \mathrm{M}, 1 \mu \mathrm{M}, 10 \mu \mathrm{M}, 100 \mu \mathrm{M}$ (3 replicates for each concentration). After shaking for $24 \mathrm{~h}, 10 \mu \mathrm{L} 5 \mathrm{mg} / \mathrm{mL}$ MTT reagent was added to each well and the plates were incubated for $4 \mathrm{~h}$ at $37^{\circ} \mathrm{C}$. Next, $100 \mu \mathrm{L} 10 \%$ sodium dodecyl sulfate (with $0.01 \mathrm{~N} \mathrm{HCl}$ ) was added to fully dissolve the crystals. Absorbance was measured at a wavelength of $570 \mathrm{~nm}$ three times, and the average values were used to construct the growth curve of cells in each group. Ratios of the $50 \%$ inhibitory concentration $\left(\mathrm{IC}_{50}\right.$ ) value in drug-resistant groups to the $\mathrm{IC}_{50}$ value of sensitive cells was considered to be the relative indicators of drug resistance in the experimental groups.

\section{MM cell autophagy detection by transmission electron microscopy}

RPMI8226/S cells and RPMI8226/DOX cells ( $2 \mu \mathrm{M}$ cells each) were centrifuged at $1000 \mathrm{rpm}$ for $5 \mathrm{~min}$ and fixed overnight in electron microscopy fixative (4\% glutaraldehyde and $1 \%$ osmic acid buffer). Cells were embedded in epoxy resin, prepared into semi-thin sections for localization, and then cut into ultrathin sections. Uranyl acetate and lead citrate staining was performed for observation under a JEM-2000EX electron microscope (JEOL, Ltd., Tokyo, Japan).

\section{Immunofluorescence}

RPMI8226/S cells and RPMI8226/DOX cells were cultured on cover slips coated with polylysine and fixed with $4 \%$ paraformaldehyde for $20 \mathrm{~min}$. Slides were washed in phosphate-buffered saline 3 times before being perforated with $0.3 \%$ Triton X-100, and then blocked with $3 \%$ bovine serum albumin at room temperature for 30 min after being washed twice with phosphate-buffered saline. Rabbit anti-human LC3 antibody (diluted 1:50 with 1\% bovine serum albumin) was added to the slides, which were then incubated in a wet box at $4^{\circ} \mathrm{C}$ overnight. Anti-rabbit secondary antibody labeled with fluorescein isothiocyanate was added to the slides, which were then incubated for $60 \mathrm{~min}$, sealed with fluorescence blocking solution, and observed under a fluorescence microscope.

\section{Detection of autophagy protein LC3 expression by Western blotting}

Separating gels and stacking gels were prepared while protein samples were extracted from RPMI8226/S cells and RPMI8226/DOX cells (at a density of $0.1 \mu \mathrm{M} / \mathrm{mL}$ ). Marker and protein samples $(50-100 \mu \mathrm{g}$ or $15-20 \mu \mathrm{L})$ were loaded into each well. Samples were transferred to a polyvinylidene fluoride membrane after electrophoresis, and the membrane was blocked with 5\% skim milk in Tris-buffered saline containing Tween 20 (10 mM Tris-buffer, $150 \mathrm{mM} \mathrm{NaCl}, 0.1 \%$ Tween 20, $\mathrm{pH} 8.0$ ). Primary antibody (anti-LC3 and anti- $\beta$-actin) was added in $5 \%$ bovine serum albumin and incubated overnight. The membrane was washed with 
Tris-buffered saline containing Tween 20 for $5 \min 3$ times. Secondary antibody was added and incubated at room temperature for $1 \mathrm{~h}$. After washing 3 times with Tris-buffered saline containing Tween 20 , the membrane was incubated with electrochemiluminescence mixture and the result was observed by developing the film.

\section{Detection of apoptosis by flow cytometry}

All procedures were conducted according to manufacturer protocols. Autophagy inhibitors ( $8 \mu \mathrm{M}$ hydroxychloroquine or $10 \mathrm{mM} 3$-methyladenine (Pan et al., 2011) were added to RPMI8226/DOX cells with DOX for $24 \mathrm{~h}$ to observe the effect of combined treatment on cells. The cells in each group were collected and adjusted to a density of $1 \mu \mathrm{M} / \mathrm{mL}$. Next, $5 \mu \mathrm{L}$ annexin- $\mathrm{V}$ and $10 \mu \mathrm{L}$ propidium iodide reagent were added to the $100 \mu \mathrm{L}$ cell suspension in microcentrifuge tubes. Additionally, $150 \mu \mathrm{L}$ binding buffer was added after the reaction in a dark room for $15 \mathrm{~min}$ and the results were analyzed by flow cytometry within $1 \mathrm{~h}$.

\section{Statistical analysis}

Data were processed using the SPSS version 11.0 software (SPSS, Inc., Chicago, IL, USA) and are reported as means \pm standard deviation. Comparisons between groups were analyzed using the independent-sample Student $t$-test, and $\mathrm{P}<0.05$ was considered to be statistically significant.

\section{RESULTS}

\section{Survival curves of DOX dose to cells}

To investigate the drug resistance effect of DOX-induced RPMI8226 cells, we applied different doses of DOX to both RPMI8226/DOX cells and RPMI8226/S cells and constructed survival curves using data obtained from the MTT assay. We observed dosage-dependent inhibition of proliferation of both cell types. The $\mathrm{IC}_{50}$ values were as follows: $\mathrm{IC}_{50}$ of RPMI8226/ $\operatorname{DOX}(8.632 \pm 2.261 \mu \mathrm{M})$ was 10.8 -fold that of RPMI8226/S $(0.798 \pm 0.514 \mu \mathrm{M})$. The results are shown in Figure 1.

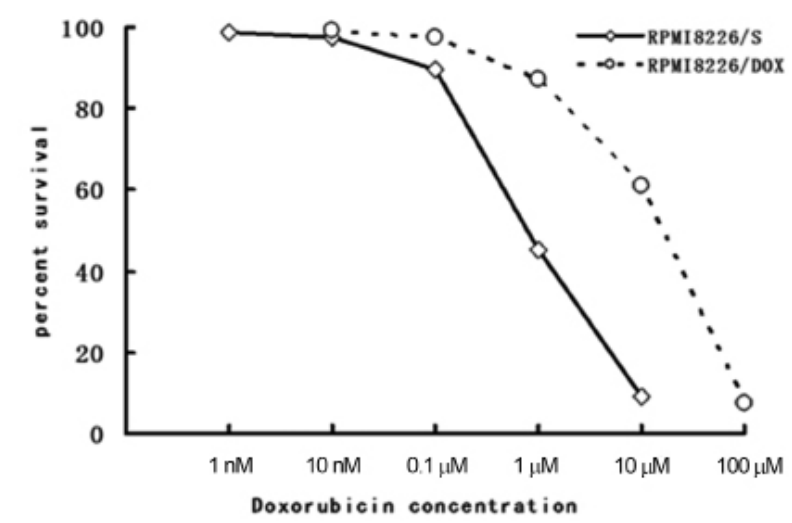

Figure 1. Effect of DOX on cell survival of sensitive (RPMI8226/S; circles) and resistant (RPMI8226/DOX; lozenges) human myeloma cells following $24 \mathrm{~h}$ of drug exposure in vitro. Data points represent the mean of 3 experiments. 


\section{Observation of autophagy of RPMI8226/DOX cells under transmission electron microscopy}

Transmission electron microscopy is the most reliable and widely used method for detecting autophagy. In this study, we observed RPMI8226/S cells and RPMI8226/DOX cells using transmission electron microscopy and found that there were fewer intracellular autophagic vacuoles in RPMI8226/S cells, indicating a low level of autophagy (Figure 2A). The autophagic response in RPMI8226/DOX cells was relatively stronger, reflected by an increase in cell volume and in the amount of autophagy and lysosomes (Figure 2B).

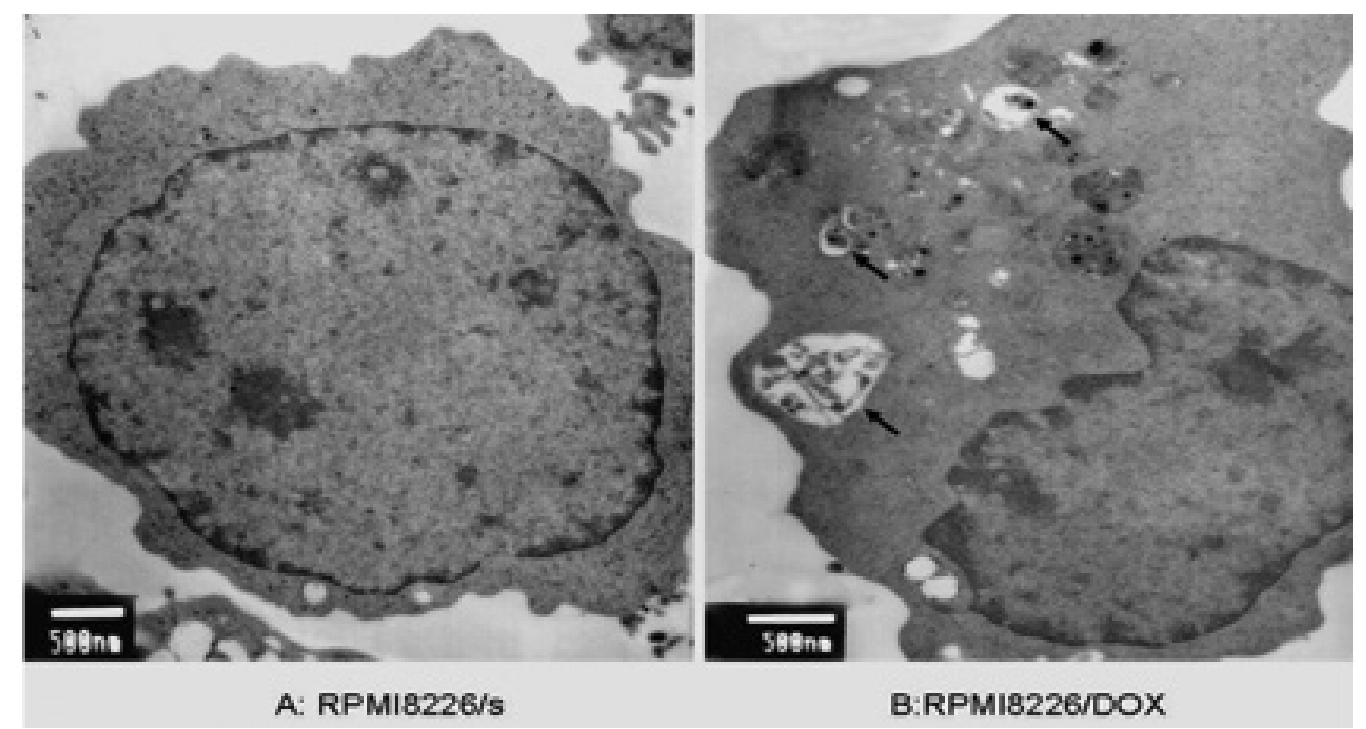

Figure 2. Autophagosome accumulation in resistant (RPMI8226/DOX) human myeloma cells by electron microscopy. Autophagic vesicles are denoted by arrows. Scale bars: $500 \mathrm{~nm}$.

\section{Observation of autophagy of RPMI8226/DOX cells by immunofluorescence}

There are 2 forms of the autophagy protein LC3 in mammalian cells: soluble cytoplasmic LC3-I and membrane-bound LC3-II. With autophagy activation, LC3-I is esterified by the binding of phosphatidyl ethanolamine, and becomes modified to the membrane-bound form of LC3-II, which is a pre-autophagy and autophagy molecular marker. Detection of endogenous LC3 by anti-LC3 antibody is superior to that using an exogenous vector of green fluorescent protein-LC3, as the latter prevents gene overexpression during transfection or the transgenic process. Changes in autophagy were observed after the staining of immunofluorescent anti-LC3 antibody, and the results showed that LC3 fluorescence in RPMI8226/S cells was diffusely distributed, while that in RPMI8226/DOX cells was in a punctate distribution, indicating the formation of LC3-II and suggesting autophagy activation (Figure 3A and B). 


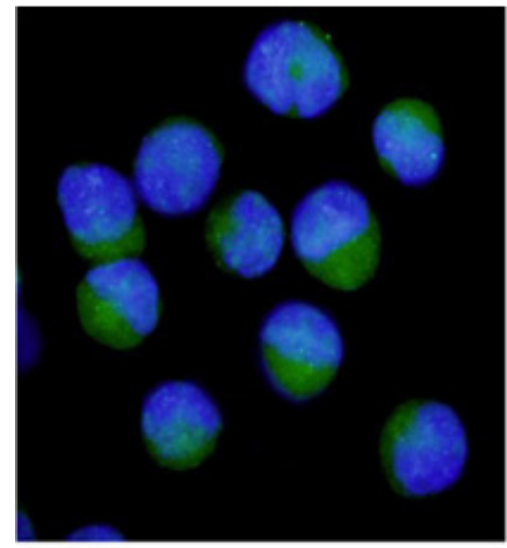

A: RPMI8226/S

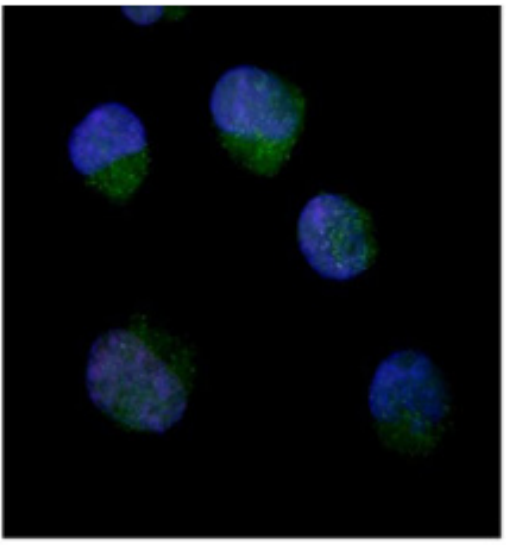

B: RPMI8226/DOX

Figure 3. LC3 relocalization in resistant (RPMI8226/DOX) human myeloma cells by fluorescence microscopy (green: anti-LC3; blue: DAPI counterstain).

\section{LC3-II expression in RPMI8226/DOX cells}

We further investigated the autophagic changes in drug resistance cells on the protein level. Western blotting analysis revealed that the expression level of LC3-II increased significantly in RPMI8226/DOX cells compared to that in RPMI8226/S cells (Figure 4).

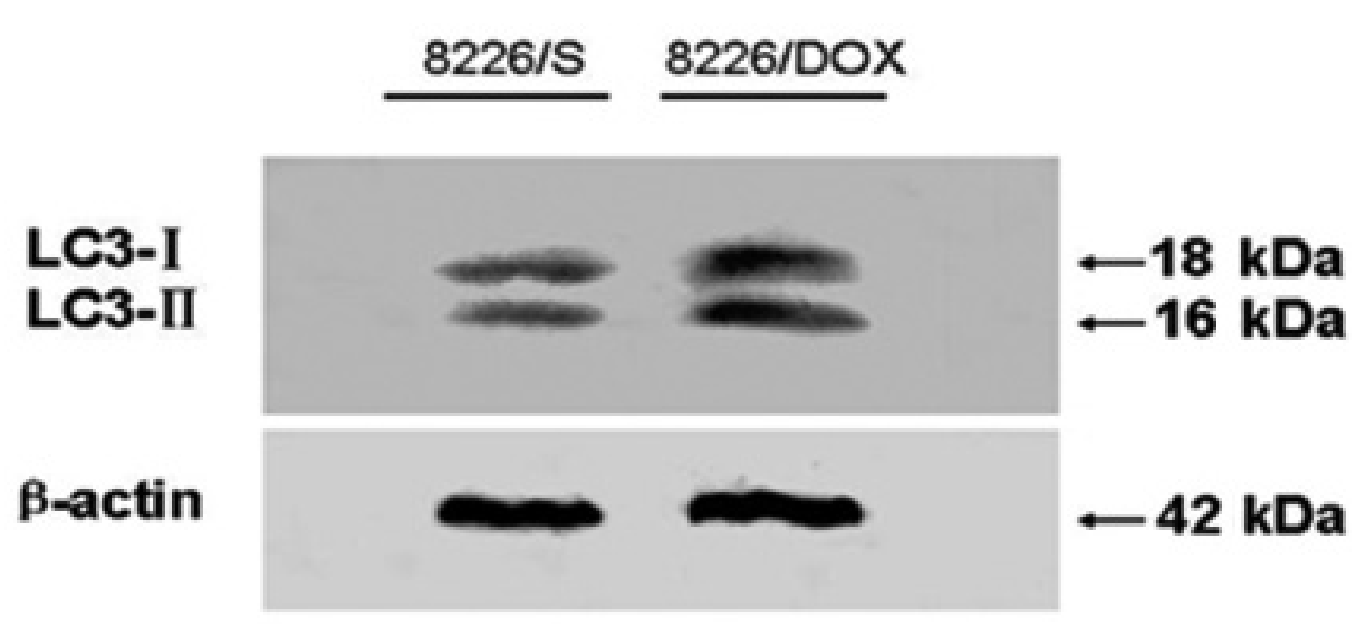

Figure 4. Expression of LC3-II in sensitive (RPMI8226/S) and resistant (RPMI8226/DOX) human myeloma cells by immunoblotting.

Inhibition of autophagy activation partially reverses drug resistance of MM cells

According to previous studies (Carew et al., 2012), when $8 \mu \mathrm{M}$ hydroxychloroquine or $10 \mathrm{mM} 3$-MA was added to inhibit autophagy, the apoptosis rate of RPMI8226/DOX cells 
processed with DOX $(2,4,6 \mu \mathrm{M})$ increased significantly compared with the rate in drugresistant cells without administration of hydroxychloroquine or 3-MA $(\mathrm{P}<0.05)$, indicating that inhibition of autophagy can partially reverse the drug resistance of RPMI8226/DOX cells (Figure 5).

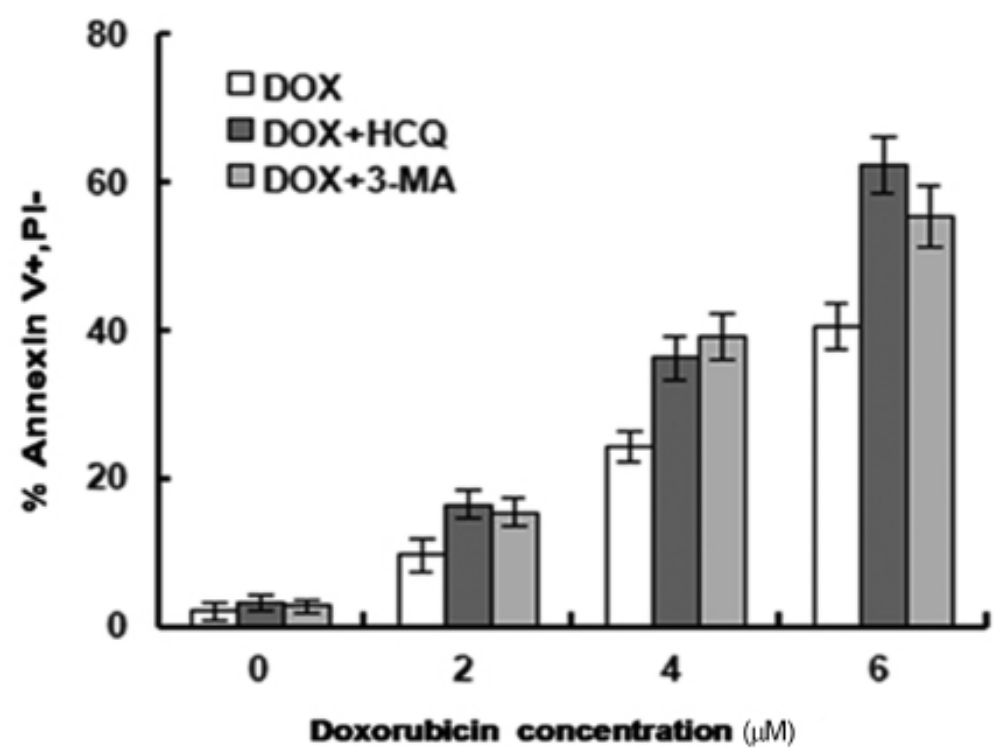

Figure 5. Effects of autophagy inhibitors on doxorubicin-induced apoptosis in resistant (RPMI8226/DOX) human myeloma cells.

\section{DISCUSSION}

A complex relationship exists between cell autophagy and tumors. Autophagy plays various roles in the different phases of tumorigenesis and tumor development; moreover, the function of autophagy varies in different tissues and cells. Liang et al. (1999) found allelic loss of Beclin1 (an autophagy-related gene) in $40-75 \%$ of sporadic human breast cancer, ovarian cancer, and prostate cancer. In the human breast cancer cell line MCF-7, expression of Beclin1 protein decreased to nearly undetectable levels. In addition, when transfected into MCF-7 cells by stable transfection, Beclin1 suppressed tumor cell proliferation in vitro and tumorigenicity in nude mice by inhibiting autophagy. In gene-targeted mouse models $(\mathrm{Qu}$ et al., 2003), mice with a single allele mutation of Beclin1 showed lower autophagy levels and higher incidence rates of various cancers, including ovarian cancer, lung cancer, hepatoma, and lymphoma, supporting the inhibitory effect of autophagy on tumorigenesis.

Numerous studies have demonstrated that autophagy combats numerous types of adverse stresses and maintains the survival of tumor cells (Li et al., 2009; Carew et al., 2012). Cytotoxic stress and metabolic stress such as hypoxia, nutrition deficiency, chemotherapy, and radiotherapy are common in tumor cells because of their high proliferative activity and high metabolism. Activation of autophagy causes ATP energy to be recycled to maintain the balance of cell metabolism. Cell apoptosis induced by chemotherapy typically accompanies high levels of cell autophagy, while genetically or medically inhibited autophagy can often increase 
cell apoptosis. These results indicate that autophagy induced by chemotherapy is cytoprotective, and the removal of internal toxic substances such as reactive oxygen species and injured organelles (such as mitochondria) may be involved in the mechanism.

Because chemotherapy-induced autophagy plays a protective role in tumor cells, there may be a correlation between autophagy and tumor cell resistance to chemotherapy. In a previous study, examining colon carcinoma, LC3 (stained with anti-LC3 polyclonal antibody) was specifically observed in tumor epithelial cells in $90 \%$ of cases, while no expression of LC3 was detected in the surrounding normal mucosa cells (Sato et al., 2007). In addition, similar LC3 distribution was observed in another study of pancreatic cancer; the prognosis of patients with high LC3 expression level was found to be relatively poor (Fujii et al., 2008). Ahn and Lee (2011) found that in NIH 3T3 cells processed with multidrug resistance gene transfection, a high level of autophagy resulted in drug resistance, while a lack of autophagy led to growth inhibition of drug-resistant NIH 3T3/Mdr cells. However, when chronic myeloid leukemia precursor cells or stem cells were exposed to imatinib, the autophagy pathway becomes activated to facilitate cell survival. Furthermore, combined application of chloroquine (an autophagy inhibitor) and imatinib significantly increased the death rate of chronic myeloid leukemia cells, even in imatinib-resistant cases (Bellodi et al., 2009). These results suggest a correlation between autophagy and chemotherapy resistance.

It is well known that in late multiple myeloma, patients often die of drug resistance. Autophagy and the ubiquitin-proteasome pathway are the major routes of protein degradation in vivo. In MM cells, hypersecretion of monoclonal immunoglobulin caused aggregation of numerous unfolded proteins, leading to endoplasmic reticulum stress and autophagy activation (Yang et al., 2011). In addition, a previous study (Pan et al., 2011) confirmed that DNAdamaging reagents such as DOX and melphalan can mediate the activation of autophagy. Based on this information, autophagy may be of great significance in the drug resistance of MM cells. Our results revealed a larger amount of autophagosome and higher expression level of autophagy protein LC3-II in DOX-resistant RPMI8226 cells compared to that in DOXsensitive RPMI8226/S cells. We also found that inhibition of autophagy can partially reverse the drug resistance of MM cells, implying that autophagy may be a major mechanism of drug resistance in myeloma. A phase I/II clinical trial regarding the effect of hydroxychloroquine combined with bortezomib in patients with relapsed/refractory myeloma is in progress.

\section{REFERENCES}

Ahn JH and Lee M (2011). Suppression of autophagy sensitizes multidrug resistant cells towards Src tyrosine kinase specific inhibitor PP2. Cancer Lett. 310: 188-197.

Bellodi C, Lidonnici MR, Hamilton A, Helgason GV, et al. (2009). Targeting autophagy potentiates tyrosine kinase inhibitor-induced cell death in Philadelphia chromosome-positive cells, including primary CML stem cells. J. Clin. Invest. 119: 1109-1123.

Carew JS, Kelly KR and Nawrocki ST (2012). Autophagy as a target for cancer therapy: new developments. Cancer Magag. Res. 4: 357-365.

Dalton WS, Durie BG, Alberts DS, Gerlach JH, et al. (1986). Characterization of a new drug-resistant human myeloma cell line that expresses P-glycoprotein. Cancer Res. 46: 5125-5130.

Fujii S, Mitsunaga S, Yamazaki M, Hasebe T, et al. (2008). Autophagy is activated in pancreatic cancer cells and correlates with poor patient outcome. Cancer Sci. 99: 1813-1819.

Li J, Hou N, Faried A, Tsutsumi S, et al. (2009). Inhibition of autophagy by 3-MA enhances the effect of 5-FU-induced apoptosis in colon cancer cells. Ann. Surg. Oncol. 16: 761-771.

Liang XH, Jackson S, Seaman M, Brown K, et al. (1999). Induction of autophagy and inhibition of tumorigenesis by beclin 1. Nature 402: 672-676. 
Pan Y, Gao Y, Chen L, Gao G, et al. (2011). Targeting autophagy augments in vitro and in vivo antimyeloma activity of DNA-damaging chemotherapy. Clin. Cancer Res. 17: 3248-3258.

Qu X, Yu J, Bhagat G, Furuya N, et al. (2003). Promotion of tumorigenesis by heterozygous disruption of the beclin 1 autophagy gene. J. Clin. Invest. 112: 1809-1820.

Sato K, Tsuchihara K, Fujii S, Sugiyama M, et al. (2007). Autophagy is activated in colorectal cancer cells and contributes to the tolerance to nutrient deprivation. Cancer Res. 67: 9677-9684.

Yang ZJ, Chee CE and Huang S (2011). The role of autophagy in cancer: therapeutic implications. Mol. Cancer Ther. 10: 1533-1541. 